\title{
Electromyography as an Important Parameter for a Proper Assessment of Dynamic Muscles Strength in Gait Analysis
}

\author{
Emiliano P Ravera ${ }^{1,2, *}$, Paola A Catalfamo ${ }^{1,2}$, Marcos J Crespo ${ }^{3}$, Ariel A A Braidot ${ }^{1}$ \\ ${ }^{1}$ Laboratory of Biomechanics, National University of Entre Ríos, Oro Verde, 3101, Argentina \\ ${ }^{2}$ National Council of Scientific and Technical Research, Buenos Aires, C1033AAJ, Argentina \\ ${ }^{3}$ Gait and Motion Analysis Laboratory, FLENI Institute for Neurological Research, Escobar, B1625XAF Argentina
}

\begin{abstract}
Normal gait is a functional movement involving the most efficient energy transfer. Cerebral Palsy describes a group of developmental disorders of movement and posture causing activity restriction or disability. It represents one of the most common cause of physical disability in children. Gait analysis provides great contributions to the understanding of gait disorders originating from musculoskeletal abnormalities. In these patients provides a mean for a more comprehensive treatment plan. At this point, the potential and necessity of using correct biomechanical models that consistently study the abnormalities become clear. Reinforcing and correcting a simple gait analysis and eliminating the unknowns when selecting the appropriate treatment are crucial in clinical settings. In this paper a musculoskeletal thigh model with a simple rescaling method using subject-specific anthropometric data is presented. The thigh model estimates muscle forces of the six muscles through the walking based in static optimization without including electromyography data as input. We used the model proposed for identify the muscles strength involved in normal and pathological gait. The forces obtaining for normal subjects achieved high concordance with their electromyography data and outcomes to other musculoskeletal model recently presented. In other hand, in patients with cerebral palsy with crouch gait the outcomes of our musculoskeletal model don't show a correct concordance with their electromyography data. For this reason we get on the conclusion that take electro myography data became important in modeling a pathological gait as crouch gait.
\end{abstract}

Keywords Musculoskeletal model, Dynamic muscles strength, Crouch gait

\section{Introduction}

Normal gait is a functional movement involving the most efficient energy transfer. Similarities between subjects are common but there are also small individual variations, so any deviation detected by comparing gait patterns are of great value.

Cerebral Palsy (CP) describes a group of developmental disorders of movement and posture causing activity restriction or disability, which are attributed to disturbances occurring in the fetal or in fant brain. It represents one the most common cause of physical disability in modern world and within the pediatrics orthopedics units[1][2][3].

Gait analysis provides great contributions to the understanding of gait disorders in CP. It allows an objective quantification of the causes of gait disorders orig inating from musculoskeletal abnormalities in patients with $\mathrm{CP}$ and it

* Corresponding author:

emilianoravera@bioingenieria.edu.ar (E P Ravera)

Published online at http://journal.sapub.org/ajbe

Copyright (C) 2012 Scientific \& Academic Publishing. All Rights Reserved demonstrates superiority to the visual or observational analysis of movement[1].

It also provides a mean for a more comprehensive treatment plan, including or excluding surgical procedures that can potentially decrease the number of surgical interventions in the life of a patient with $\mathrm{CP}[4][5][6]$.

In general the abnormal gait pattern of patients with $\mathrm{CP}$ are the result of primary problems (as deficient selective motor control, abnorma lities of balance and abnormal central nervous system tone drive); secondary abnormalities characterized as growth disorders (for example growth of bone and muscles growth) and tertiary abnormalities as those compensations that the individual uses to circumvent the other abnormalities of gait [5].

So, much of the difficulty encountered in studying pathologic gait involves the separation of the true pathology. However, good treat ment demands their separation because, to optimize the efficiency of gait, we must correct the former and not interfere with the latter[7].

Thus, the models could improve recommendations for orthopaedic surgery, based on a quantitative description of how to modify muscle force generation and how these 
modifications affect the action of the muscles during crouch gait[8][9].

One of the most common abnormalities in children with $\mathrm{CP}$ is crouch gait, characterized by excessive knee flexion during the terminal swing phase and initial loading response, and also increased dorsiflexion during the whole gait cycle. However, it is thought that this abnormality may be due to a variety of neuromuscular disorders which are not easily detectable.

Crouch gait is commonly treated by a combination of orthopaedic procedures with the objective of diminishing the excessive knee flexion and imp roving the walking pattern[10] [11][12].

So, this pathological gait needs to be treated since the flexion angle at the knee and hip joint tend to increase with age, due to increased body weight, which compromises the ability for independent walking[11].

Methods of treatment to decrease spasticity allows the child to have greater range of motion, less spastic response to stretch, and better potential to develop using voluntary muscle activity during gait. So, surgical procedures to muscle lengthening are used but insufficient muscle strength can be a major cause of ongoing disability[5].

However, the causes of an appropriate treatment for gait abnormalities are difficult to determine because the movements generated by muscular forces of patients are not completely resolved and clearly understood yet[13][14]. Hence the explicit identification of the anomaly does not necessarily present a direct solution[15].

Due to the mechanical redundancy displayed by the human locomotor system, activities such as walking can be performed in different ways and in many combinations of muscle forces wh ich can generate the same net joint mo ment. However, there are similarities in the muscle activation patterns (MAP) of different people performing the same well-learned task[16]. Experimental measurements of muscle forces during loco motion of animals showed that the MAP are in general stereotyped[17].

These consistencies suggest that the central nervous system uses specific principles for the control of individual muscle forces, and that principles are the same for different persons. That leads to the hypothesis that central nervous systemselects optimal MAP with a set of criteria that are still unknown[16].

Assuming that the central nervous system minimizes stress on the muscles in the body, leads to the hypothes is that muscle strength may be found as the solution to an optimization problem.

A treatment plan is further complicated because there is not clear scientific basis to determine how the neuro-muscular-skeletal impairment contributes to the abnormal movement[18]. Abnormalities of the knee may be related to dynamic conditions such as spasticity, secondary static muscle contractures of the hip, knee and ankle, or a combination of short and spastic muscles.

A correct determination of the etiology of abnormal patterns of the knee is the key to select the appropriate therapy[19], presenting a major challenge at present since there is not sufficient theoretical basis to determine the biomechanical causes of abnormal gait of these patients [20] [21][22].

At this point, the potential and necessity of using correct biomechanical models that consistently study the abnormalities become clear. Reinforcing and correcting a simple gait analysis and eliminating the unknowns when selecting the appropriate treatment are crucial in clinical settings[23].

The main limitations of the musculoskeletal models are that,

a) they are anatomically and physiologically incomp lete;

b) they show insufficient accuracy in the estimation of the used parameters;

c) it is difficult to validate them.

A musculoskeletal thigh model with a simple rescaling method using subject-specific anthropometric data to fit the parameters is proposed in this study. The model estimates muscle forces from six muscles of the thigh during walking. The model uses a quadratic approximation method algorithm to find the solution of a non-linear mathematic program based on static optimization.

Electro myography of each subject under analysis was use to correlate it with the muscles strength modeled as a validation procedure for the outcome of the proposed musculoskeletal model. Also, the outcomes of the presented model from unimpaired subjects were compared with results published in the literature.

Finally, the model was used to identify muscles strength involved in pathological gait of CP patients who presented crouch gait.

\section{Method}

\subsection{Participants and Procedure}

$\mathrm{CP}$ and control group (CG) were recruited for the study. The study included a first group of 10 ambulatory patients with CP, age 8-23 years, $1.50-1.94 \mathrm{~m}$ in height and 49-105 $\mathrm{kg}$ in weight and the second group included five unimpaired subjects, age 7-11 years, $1.24-1.67 \mathrm{~m}$ in height and 22-53 $\mathrm{kg}$ in weight (Table 1).

Table 1. Description of participants, age, height, weight and spatiotemporal gait parameters, all presented as mean ( $\min -\max )$

\begin{tabular}{|c|c|c|c|}
\hline & CP n=20 trial & CG n=10 trial & $\mathrm{p}$-Value \\
\hline Age (years) & $13.5(8-23)$ & $9.8(7-14)$ & 0.1172 \\
\hline Height $(\mathrm{m})$ & $1.45(1.08-1.83)$ & $1.42(1.24-1.67)$ & 0.7463 \\
\hline Weight $(\mathrm{kg})$ & $42.3(25-79)$ & $36.6(22-53)$ & 0.5315 \\
\hline Velocity $(\mathrm{m} / \mathrm{seg})$ & $0.97(0.55-1.35)$ & $1.10(0.97-1.28)$ & 0.6527 \\
\hline Stride length $(\mathrm{m})$ & $0.97(0.66-1.38)$ & $1.11(1.07-1.18)$ & 0.0001 \\
\hline Cadence steps/min & $121(103-141)$ & $117.2(105-133)$ & 0.7446 \\
\hline Stance phase $(\%)$ & $60.83(54-68)$ & $58.5(57.5-59.5)$ & 0.6839 \\
\hline
\end{tabular}

Several parameters are becoming the standard description of the gait of children with $\mathrm{CP}$ as it enables clinicians and researchers to have a common language[24]. 
Gross Motor Functional Classification Scale (GMFCS), provides a means of describing children's locomotion function ranging from running and walking independently to requiring assistance to move a wheelchair. The Functional Mobility Scale (FMS) to further classify children who are ambulatory based on their walking ability at 5 meters, 50 meters, and 500 meters.

The Functional Assessment Questionnaire (FAQ), ask children and their caregivers a variety of questions aimed at determining the child's ability to walk short distances as well as manage common architectural barriers.

Another tool, approaches using quantified motion analysis. The Gillette Gait Index (GDI), is a system of normalcy of gait using deviations from the normal walking patterns derived from three-dimensional gait analysis.

By combing, the qualitative and quantitative information that show these parameters provide a clear picture of the ambulatory capabilities of these patients, see Table 2 .

Table 2. Generals functional characteristics of gait patterns of patients

Patient
\begin{tabular}{|c|c|c|c|c|}
\hline \multirow{2}{*}{ Patients } & \multicolumn{4}{|c|}{ Functional Range } \\
\cline { 2 - 5 } & FAQ & GMFCS & FMS & GDI (right/left) \\
\hline 1 & 7 & II & 6.5 .5 & $75.39 / 71.46$ \\
\hline 2 & 9 & II & 6.6 .5 & $72.85 / 68.10$ \\
\hline 3 & 8 & II & $6.5 . N$ & $64.21 / 69.04$ \\
\hline 4 & 8 & II & 6.6 .5 & $68.80 / 68.98$ \\
\hline 5 & 8 & III & 5.5 .4 & $62.12 / 67.61$ \\
\hline 6 & 9 & II & $5.4 . N$ & $60.45 / 62.78$ \\
\hline 7 & 9 & I & 6.6 .6 & $71.99 / 78.79$ \\
\hline 8 & 9 & I & 6.6 .6 & $66.39 / 72.51$ \\
\hline 9 & 10 & II & 5.5 .5 & $71.27 / 71.54$ \\
\hline 10 & 9 & II & 6.6 .5 & $67.45 / 71.55$ \\
\hline
\end{tabular}

The selection criteria for patient with $\mathrm{CP}$ were : Gross Motor Function Classification System (GMFCS) levels 1, 2 or 3 (a mbulant without walking aids), no orthopaedic surgery or botulinum toxin treatment within the last 6 months, diagnosis of symmetric Spastic Diplegia (SD) and walked with crouch gait ( $\geq 25$ degree of knee flexion in mid stance).

Data from both, healthy and CP populations were provided by the Gait and Movement Laboratory at FLENI Institute for Neurological Res earch, Escobar, Argentina. The Hospital Research Ethics Committee reviewed and approved this study. The protocol was explained to the subjects and a consent form was signed by the subjects or their carers.

\subsection{Data Collected}

Kinematic data was recorded by a motion capture system (Elite 2002 BTS Bioengineering, Italy) with 8 different cameras $(100 \mathrm{~Hz})$ and two force plates (Kistler 9281E, Kistler Group, Switzerland). Twenty two retro-reflective skin markers were placed over bony landmarks (as indicated by the Davis protocol[25]). After measurement, all data was imported into MATLAB (MathWorks, Natick, MA). Marker trajectories were filtered with a zero-lagged Butterworth filter with a cutoff frequency of $6 \mathrm{~Hz}$ and order 8 .

Muscles electrical activity data was recorded using surface dynamic electromyography (Teleemg BTS Bioengineering, Italy). The eight channels of acquisition were used with a sampling frequency of $2000 \mathrm{~Hz}$ for normal subjects and patients with $\mathrm{CP}$.

Electromyography data was rectified and filtered with a low-pass Butterworth filter with a cutoff frequency of $6 \mathrm{~Hz}$ and order 2, and their peak value was used for normalization.

Anthropometric measurements (height, weight, leg length, knee jo int width and distance between anterior superior iliac spines (ASIS)) for each participant were recorded by an experienced physiotherapist.

\subsection{Musculos keletal Model}

Figure 1 show the musculoskeletal mechanical system proposed in this study. This model consists of three segments (pelvis, femur and tibia) and six muscles.

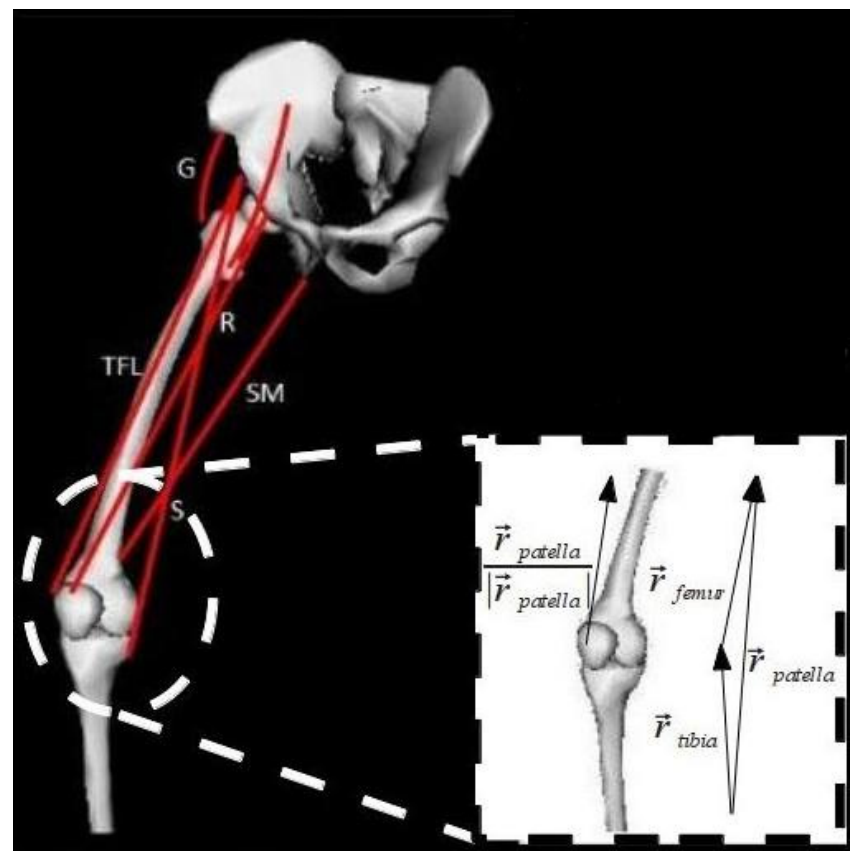

Figure 1. Scheme of musculoskeletal thigh model

Four of the muscles were considered biarticular: Tensor Fascia Lata (TFL), Semimembranosus (Semi), Sartorius (S) and Rectus Femoris (RF) and two were modeled as monoarticular: Gluteus (G) and Iliac (I). The model has six degrees of freedom (DoF), three for the hip joint and three for the knee.

The selection criteria used for selecting the muscles included in our musculoskeletal model were:

- Antagonistic muscles with biarticular actions, and major physiological cross section area (PCSA).

- Monoarticular muscles such as the gluteus and iliac because they have great importance in the movement of the hip.

The model used estimations of the patches of origin and insertion and the directions of the muscles were assumed to be linear between the origin and insertion sites, see Table 3. 
The positions of the patches of the six muscles were used from the estimated ones by using cadaveric references and axis systems embedded on and lin ked to the movement of the pelvis and the leg respectively[26].

The multi segment model used in this study was based on a simp le rescaling method, by adjusting the parameters using subject-specific anthropometric data.

Table 3. Musculoskeletal morphological parameters. Patch estimation as percentages of the anthropometric data (ASIS length or knee diameter). $\left({ }^{*}\right)$ Proportional to the height, $(* *)$ Mid position between anterior and posterior iliac superior spine, $(* * *) 7 \%$ within the origin of the gluteus

\begin{tabular}{|c|c|c|c|c|c|c|c|}
\hline & PCSA & \multicolumn{3}{|c|}{ Origin (\%) } & \multicolumn{3}{c|}{ Insertion (\%) } \\
\cline { 3 - 8 } & $\mathrm{cm}^{2}$ & $\mathrm{X}$ & $\mathrm{Y}$ & $\mathrm{Z}$ & $\mathrm{X}$ & $\mathrm{Y}$ & $\mathrm{Z}$ \\
\hline $\mathrm{S}$ & 8.8 & 11.4 & 26.6 & 12.5 & 31.3 & 17.7 & 3.7 \\
\hline $\mathrm{RF}$ & 28.9 & 11.4 & 13.3 & 6.3 & 35.2 & 0.0 & 0.0 \\
\hline $\mathrm{TFL}$ & 5.9 & 11.4 & 26.6 & 12.5 & 3.0 & 15.2 & 20.8 \\
\hline Sem & 15.75 & 22.2 & 18.2 & 0.0 & 21.3 & 17.7 & 3.7 \\
\hline $\mathrm{G}$ & 49.35 & $* *$ & $* *$ & $* *$ & 31.2 & $6.3 *$ & 15.5 \\
\hline $\mathrm{I}$ & 16.25 & $* * *$ & $* * *$ & $* * *$ & 31.2 & $8.3 *$ & 21.5 \\
\hline
\end{tabular}

For calculate the time-varying muscle force vectors a line action is assumed. The model assumes that the force transmission by the muscle acts along a straight line connecting the points of origin and insertion. So, the equation (1) defined $\vec{\tau}_{i k}$ as the unit muscle force vector for the ith muscle[26].

$$
\vec{\tau}_{i k}=\frac{\vec{r}_{o / i}}{\left|\vec{r}_{o / i}\right|}=\frac{\vec{r}_{i}-\vec{r}_{o}}{\left|\vec{r}_{i}-\vec{r}_{o}\right|}
$$

A single straight-line model between the anatomical origin and insertion is not adequate for the Rectus Femoris because the patella acts as an important lever in the knee joint. In this case the insertion is re-calculated as the difference between a vector fixed to the tibia and another fixe to the femur (Figure 1). Mathematically the direction of real action is calculated as seen in (2),

$$
\vec{r}_{\text {patella }}=\frac{\vec{r}_{\text {tibia }}+\vec{r}_{\text {femur }}}{\left|\vec{r}_{\text {tibia }}+\vec{r}_{\text {femur }}\right|}
$$

The time-varying muscle mo ment may be expressed as the mo ment generated by a unit force (3).

$$
\vec{m}=\vec{r}_{i} \times \vec{\tau}_{i k}
$$

where $\vec{m}$ is the moment associated with the $i t h$ muscle and $\vec{r}_{i}$ is the location of $i t h$ muscle origin/insertion with respect to the joint center[27].

\subsubsection{Individual Muscle Strength Dynamics}

We define muscle strength dynamics as forces that are developed for the individual muscles throughout the gait cycle.

A static optimization was used to calculate the muscle strength for each sample independently (4). Thus, muscle forces are constrained by two physiological limitations: muscles may provide only contraction forces and these forces are limited to a maximum.

$$
\operatorname{Min}_{f} G\left(f_{i}^{(m)}\right)
$$

restric

$$
\begin{array}{cl}
\sum_{i=1}^{6} f_{i}^{(m)} \vec{\tau}_{i k} \leq \vec{F}_{k} & ; k=1,2 \\
\sum_{i=1}^{6} f_{i}^{(m)}\left(\vec{r}_{i} \times \vec{\tau}_{i k}\right) \leq \vec{M}_{k} & ; k=1,2 \\
f_{i}^{(m)} \geq 0 & ; i=1,2, \cdots 6
\end{array}
$$

were $\vec{F}_{k}$ and $\vec{M}_{k}$ are the intersegmental joint force and net joint moment and $f_{i}^{(m)}$ is the norm of ith muscle.

The Optimization problem have inequality constraints because only a limited group of muscles of the thigh were modeled and also the forces developed by ligaments and cartilage of the joints were neglected.

The optimization problem was solved through the MATLAB Optimization Toolbox. The "fmincom" function that finds a constrained minimum of a scalar function of several variables starting at an initial estimate was used. This is generally referred to constrained nonlinear optimization or nonlinear programming (NLP).

The algorithm used in solving the NLP is the successive quadratic approximations, which is based on successive approximations to the Lagrangian function. Approximation to the Hessian matrix is given by the method of Broyden-Fletcher-Goldfarb-Shanno (BFGS) and using the approximation of Sherman-Morris on-Woodbury leads to the expression of the inverse of the Hessian (5).

$$
B_{k+1}=B_{k}-\frac{B_{k} s_{k} s_{k}^{T} B_{k}}{s_{k}^{T} B_{k} s_{k}}+\frac{y_{k} y_{k}^{T}}{y_{k}^{T} s_{k}}
$$

were $s_{k}=\vec{x}_{k+1}-\vec{x}_{k}$ and $y_{k}=\nabla L\left(\vec{x}_{k+1}\right)-\nabla L\left(\vec{x}_{k}\right)$. The vector $\vec{x}_{k}$ represents the forces at the time $k$, whose elements are the norm of muscles strength $f_{i}^{(m)}$. The vector $\nabla L\left(\vec{x}_{k}\right)$ is the gradient of the Lagrangian function at the time $k$.

This method is considered one of the more effective quasi-Newton methods, showing an overall superlinear convergence, high robustness in problems where the objective function is nonlinear, satis fying the secant rule and ensuring that the Hessian matrix is positive semidefinite in resolution quadratic problem[28].

Among the objective functions used in static optimization in gait, currently the use of polynomials criteria stands up.

A polynomial optimization function of third order with normalization factor which in this case was Muscle PCSA was implemented (6). The physiological behavior behind this criterion is to minimize muscle fatigue[16].

$$
G\left(f_{i}^{(m)}\right)=\sum_{i=1}^{6}\left(\frac{f_{i}^{(m)}}{P S C A_{i}}\right)^{3}
$$


This static optimization problem was restricted with the tridimensional net total torque of the six muscles actuating on the joints of the knee and hip.

Polynomial functions may describe physiological conditions. They require the use of complementary restrictions that prevent individual muscle forces from exceeding their physiological maximum when the external load increase in normal subjects, or under abnormal or pathological conditions of internal loads. As pathological conditions of internal load are expected in patient with SD $\mathrm{CP}$, muscular forces were restricted by two physiological limitations:

- Muscles only can develop contraction forces.

- These contractile forces have a maximum physiological limit.

The cost functions used in optimization problems that represent muscle fatigue and metabolic cost as the polynomial, can predict: (i) the reciprocal coactivation of one-joint antagonist muscles; (ii) the coactivation of one-joint synergists with their two-jo int antagonist; (iii) the simultaneous activation of synergists crossing the same joints and (iv) a strong relationship between force and activation of two-joint muscles and moments at the two joint[16].

\section{Results}

The net joint moments of patients and CG obtained from the link model segment are within the values shown in the literature (Figure 2)[5][28].
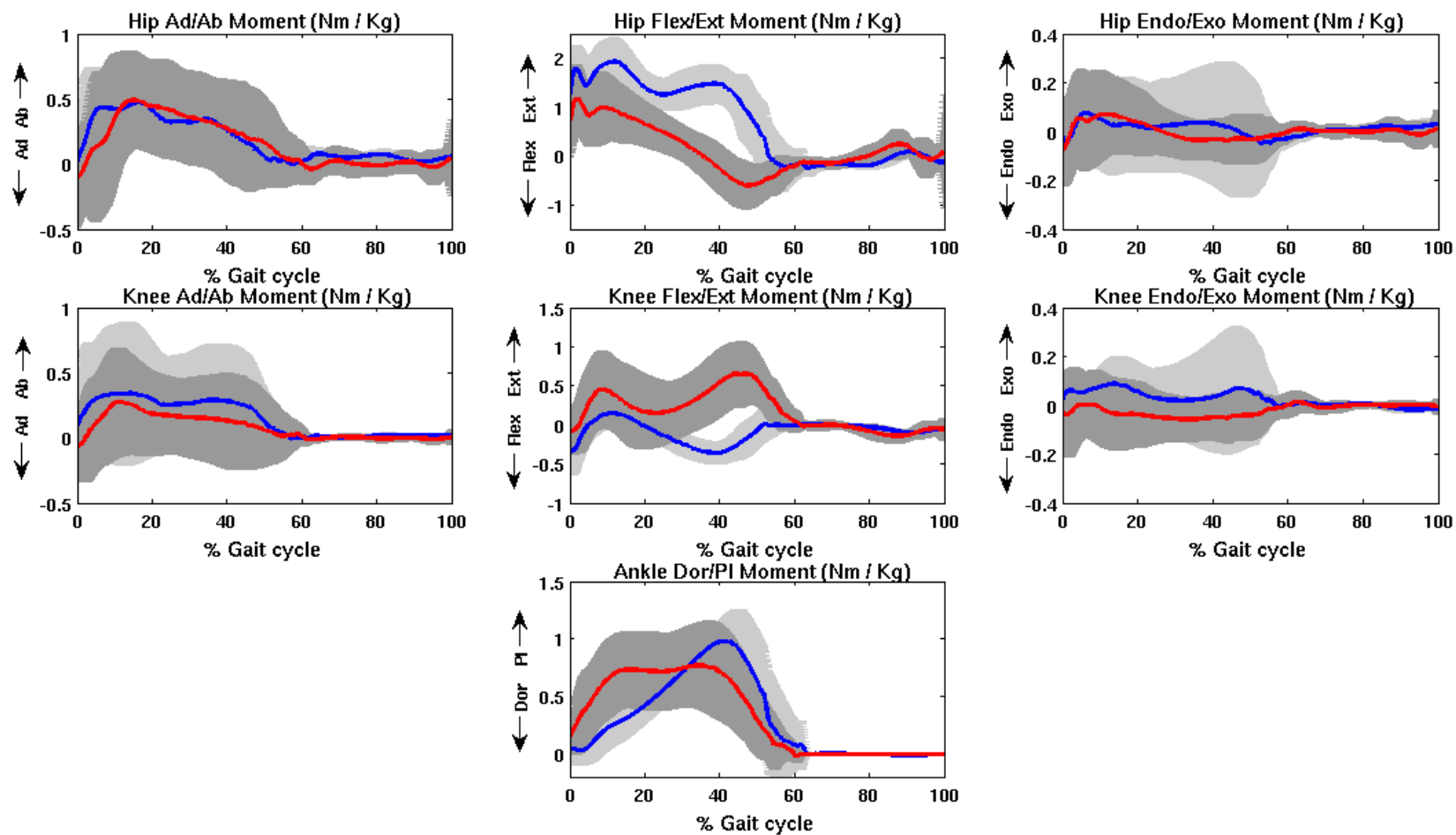

Figure 2. Mean and standard deviation of moment of hip, knee and ankle joints of patients with CP (red line) and normal subjects (blue line)
These results are used as input for the static optimization problem in the healthy subjects and patients with CP.

Figure 3 shows the norm of muscle forces throughout the gait cycle found by our model for subjects with and without pathological movement.

This model shows a good relationship between the data of electro myography activity in different muscle groups and the estimation of dynamics muscle strength for unimpaired subjects, see Figure 4.

Also seen in Figure 4 that the model respects the areas where these muscles are very important in driving. The results of the model show that Semimembranosus muscle is ma inly activated during stance and at the end of swing phase, which coincides with the literature[29].

Figure 5 shown that our model presents good concordance in the results observed in healthy subjects recently presented by other authors[8], in areas where forces have achieved their maximum level.

The main differences between the two models are given in the Rectus Femoris (Figure 5). Van der Krogt et al.[8], show that in the end of stance phase there is a peak above $80 \%$ of maximumvalue. This is not observed in the present model.

However, the model proposed in this study shows that the Rectus Femoris presents a peak of strength at the end of the swing phase. In the other hand at the same moment the Rectus Femoris contracts in combination with its antagonis tic muscles that result in a soft extension knee jo int, which coincides with the electromyography data recorded (Figure 4). 

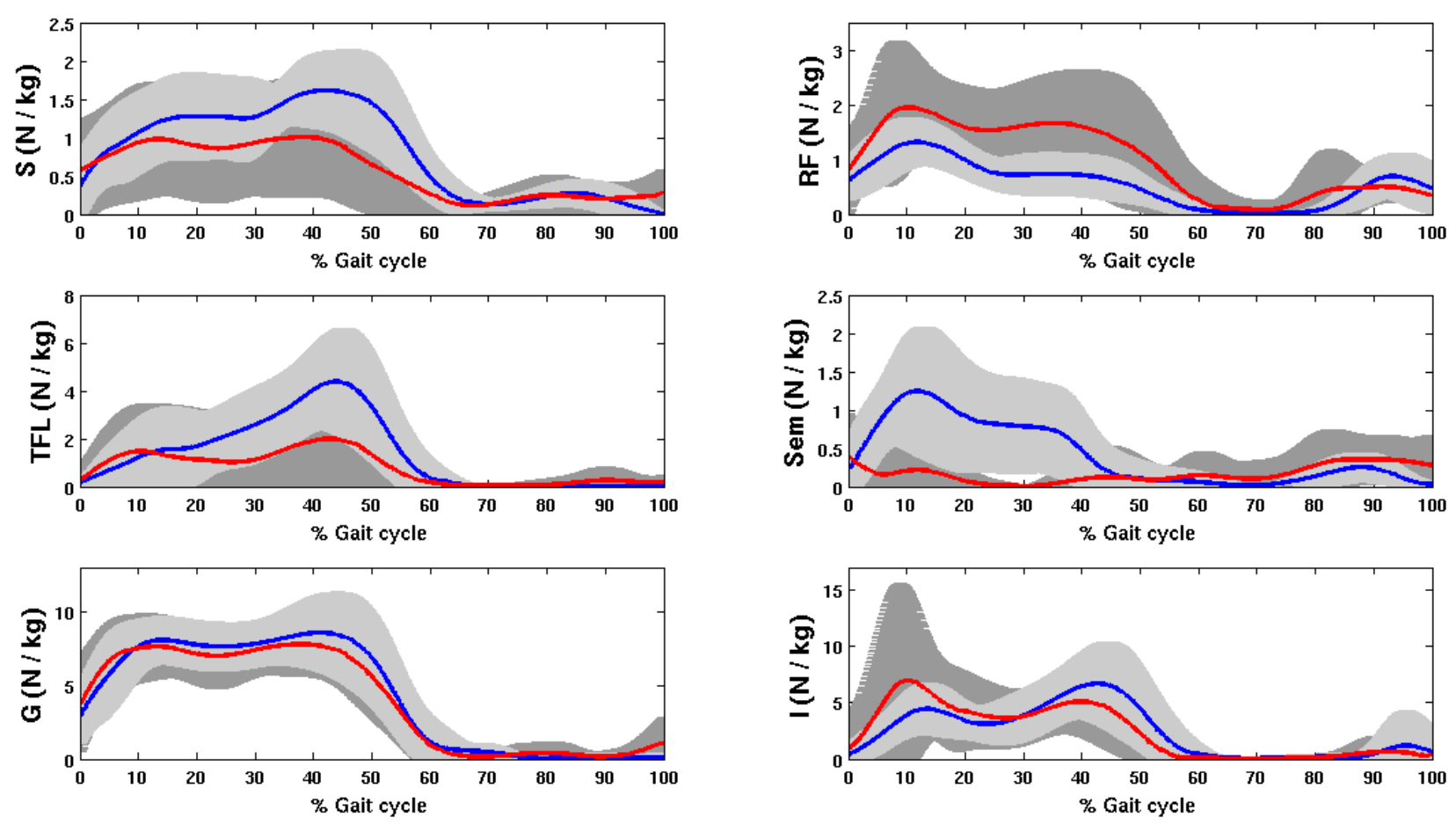

Figure 3. Mean and standard deviation of Norm of muscles strength dynamics for healthy subjects and patient with crouch gait
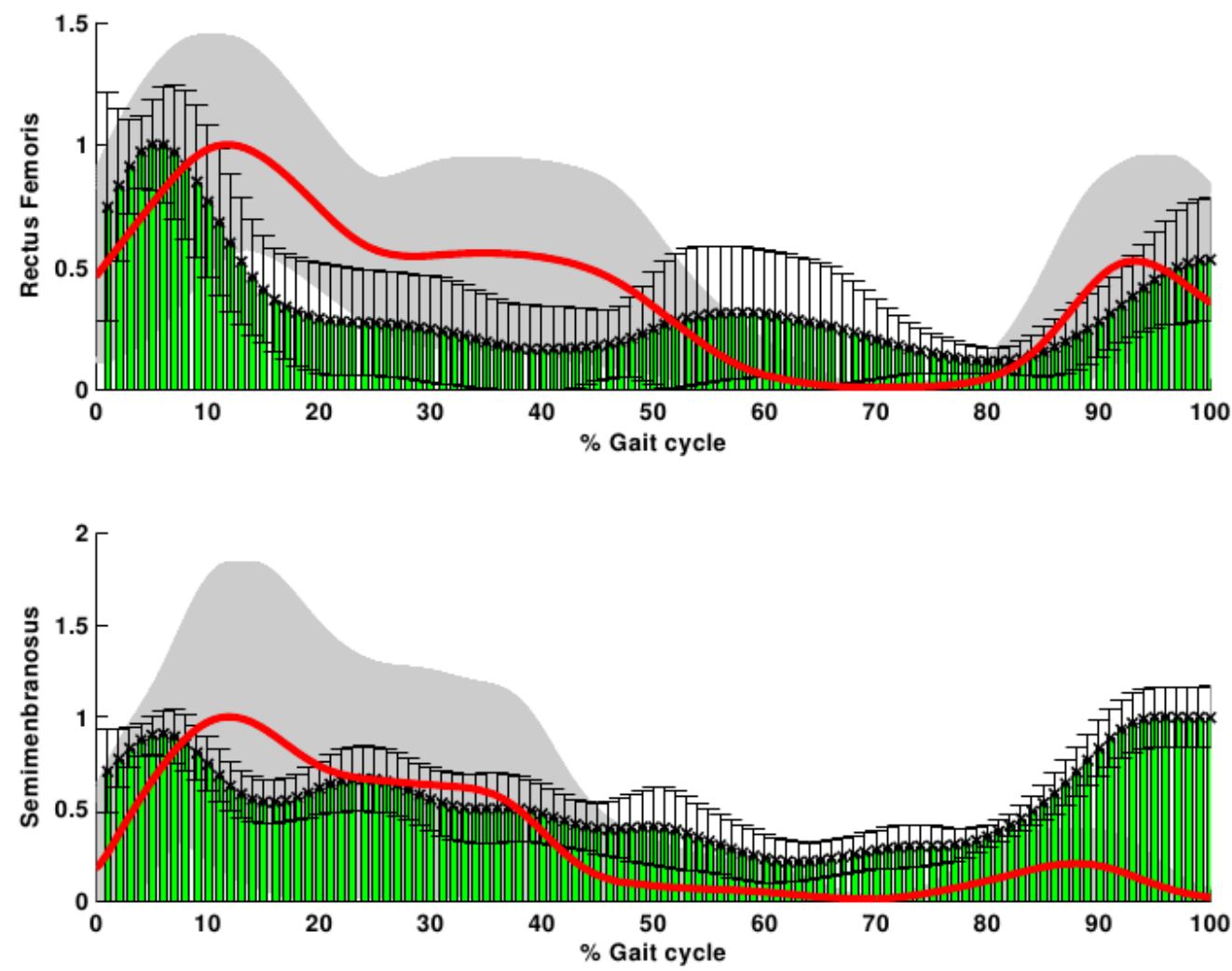

Figure 4. Norm of muscle strength and electromyographic activity of healthy subjects. Rectus Femoris and Semimembranosus (red line- grey shadow) versus the EMG (green-black bars) normalized to their maximum 

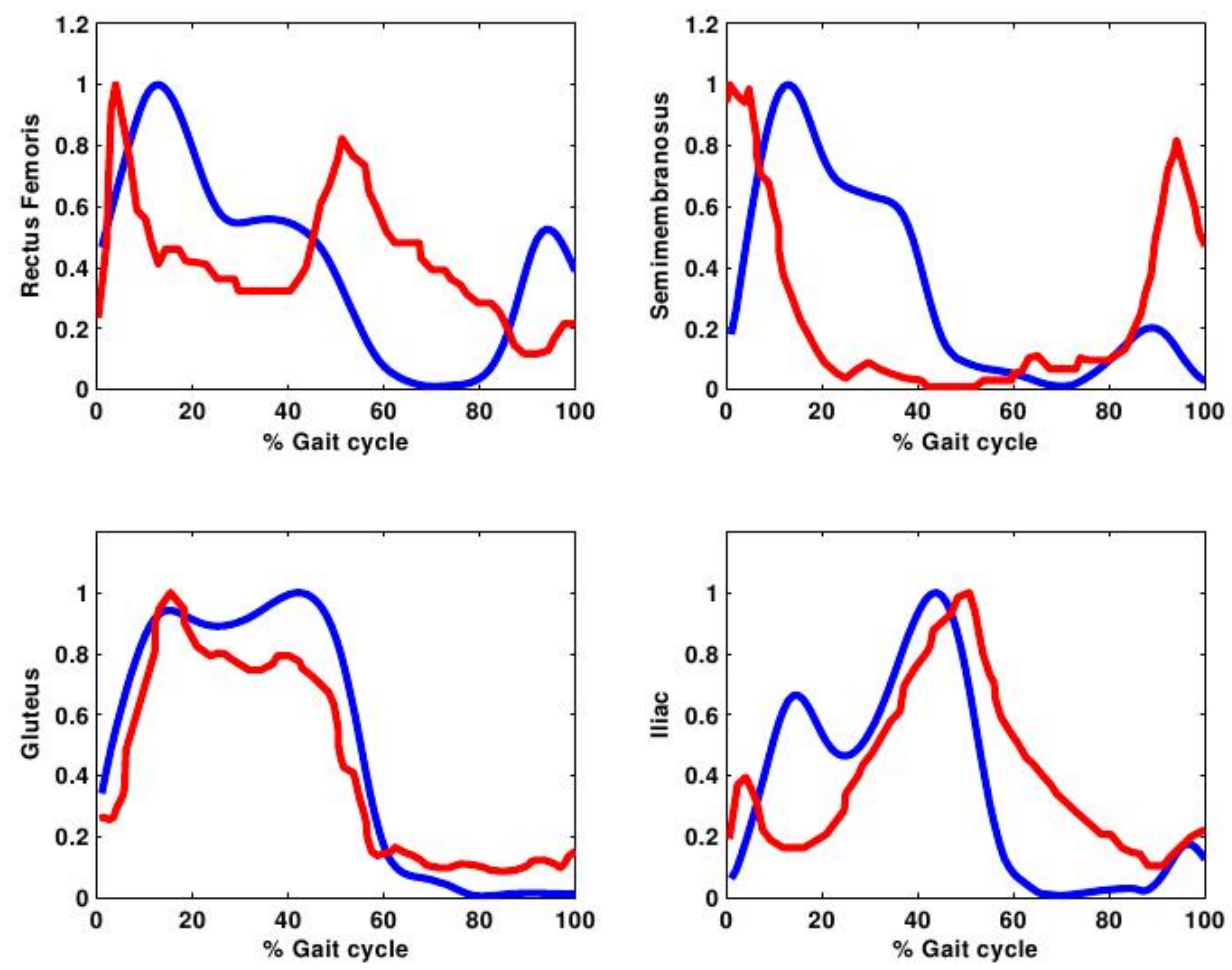

Figure 5. Norm of muscle forces throughout the gait cycle compared with the results observed by other musculoskeletal model [8]. These results are normalized to the maximum value for comparison. Blue lines represent our model and Van der Krogt et al. model in red lines

Figure 6 shows the mean and standard deviation of muscles strength dynamics of the Rectus Femoris and Semimembranosus throughout the gait cycle obtained by the model and the electromyography data for patients with crouch gait.
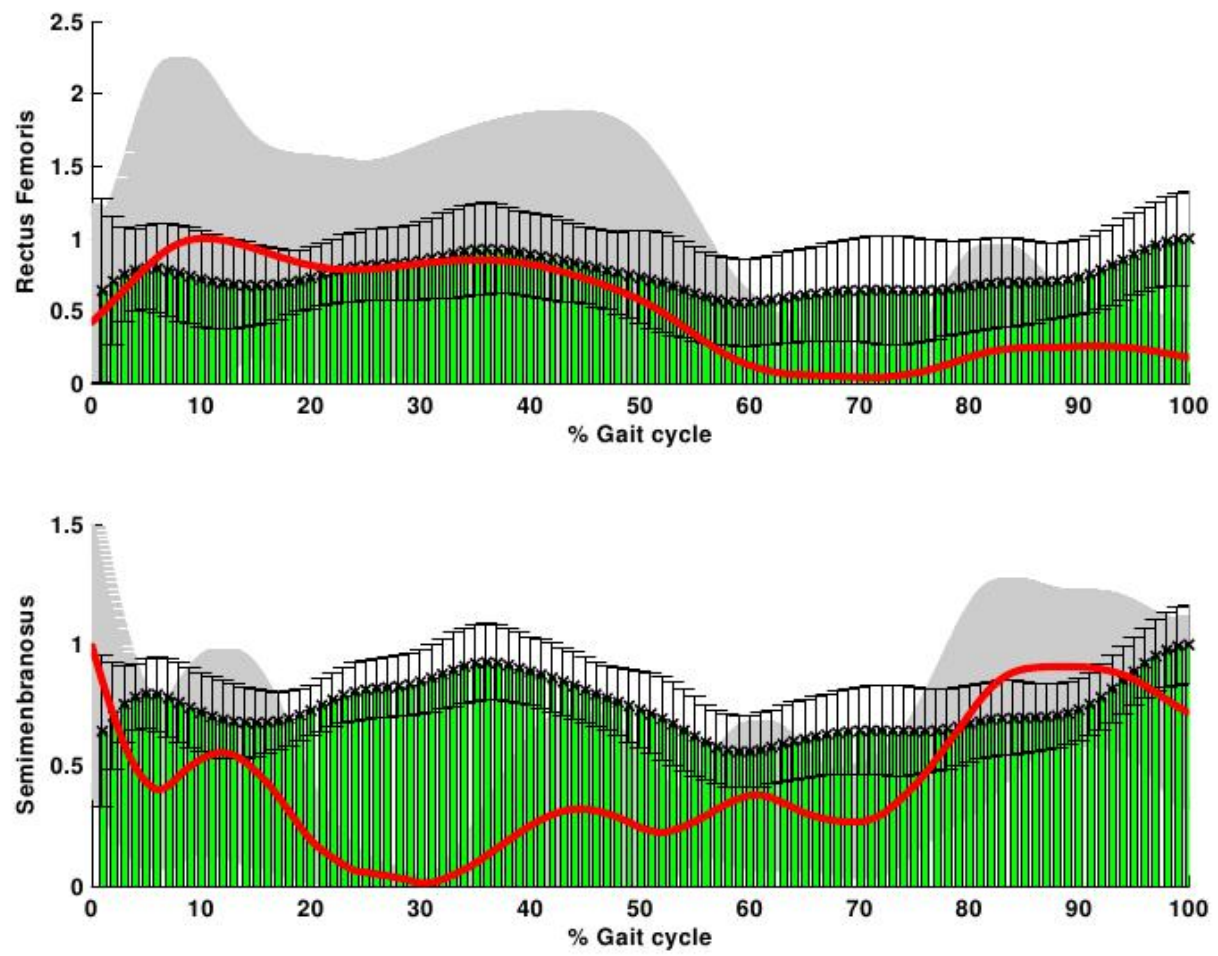

Figure 6. Norm of muscle strength and electromyographic activity of patients with CP. Rectus Femoris and Semimembranosus (red line- grey shadow) versus the EMG (green-black bars) normalized to their maximum

We see that in spite of the similarities shown between these relationships decreased in patients with neurological muscle strength dynamics and EMGs data in healthy subjects, diseases such as CP who develop crouch gait. 
An increase respect $\mathrm{CG}$ in the knee extensor moment may be due to an increase in the passive force generated by the hamstrings (Figure 2)[3]. A knee flexion during all phases of the patient's gait may have been caused by a co-contraction of the Hamstrings and Rectum Femoris.

From these results, it is possible to see that the muscle groups that act during the flexion of knee joint, such as Hamstrings and Tensor Fascia Lata were decreased in $\mathrm{CP}$ patients respect to $\mathrm{CG}$ and the strength of Rectus Femoris was increased throughout the gait cycle (Figure 3). Also, as shown in the Figure 6, the muscles strength dynamics of this patient's not present a good similitude with their EMGs data recorded.

These results lead to be noted that in patients with gait disorders this parameter can be critical for a determination of the accurate value of muscles strength through the gait cycle.

\section{Conclusions}

Information about level and distribution of dynamic muscle strength in unimpaired subjects and children with $\mathrm{CP}$ would help to better understand the action of the musculoskeletal system that generates the net mo ment jo ints.

One limitation of this model is the limited number of modeled muscles. Also, it has been assumed that each muscle generate forces only in the direction of a single fiber that is in the center of the muscle.

Electromyography data was not used for limiting the timing of the activity of the muscles. This simplification was adopted because it is not possible to record the electro myography signal from all modeled muscles in this work. However, the recorded signals (EMGs) of several muscles were compared with the dynamics muscle strength estimate by the model.

For the unimpaired group, the lower limb strength calculated in all modeled muscle groups presented a stereotyped morphology, as seen from the similar morphology presented by all healthy subjects (Figure 3 ), in accordance with previous studies [16].

Also, for this group, muscle forces presented expected physiological features such as a delay between dynamic strengths and EMG data recorder. This delay is related to the fact that total strength is the sum of the recruitment of motor units, and the motor unit action potential reaches the maximum 40 to 100 milliseconds later to the electrical activation recorder[24].

The forces obtained by this model achieved high concordance with the electromyography data and other musculoskeletal mode1[8]. Hence, it is considered that the forces estimated for healthy subjects by this model consistently approximate the actual forces acting in hip and knee jo ints

Hicks et al.[13], demonstrated that crouch gait reduces the capacity of muscles to extend the hip and knee joints in stance phase. As a consequence, muscles must work harder to maintain a particu lar limb position, which helps to explain the increase in energy expenditure when walking in a crouched posture.

Also previous simulations indicate that crouch gait is characterized by larger muscle forces due to support body weight and propel the body forward throughout single-limb stance[30].

Our musculoskeletal model showed only an increased strength in the Rectus Femoris and a decrement on the other modeled muscles, but little similarity with EMGs data (Figure 6) in contrast with these facts. This may be related to the fact that in this model does not take account the electro myography as an input parameter to restrict the value of dynamics muscles strength.

For this reason we get on the conclusion that take electromyography data became important in modeling a pathological gait as crouch gait.

So, the inclusion of electromyography data in the modeling of muscles strength behavior in patients with pathological gait as CP will help to look for the increases in the co-contraction associated with their degree of spasticity of some muscles.

However, despite of the simplicities that present our model, in the number of modeled muscles and the lack of EMGs data; in healthy subjects there were satisfactory results. It demonstrates that in these subjects the EMG is not a critical parameter to include in the musculoskeletal model to find the dynamics muscles strength.

This study showed a musculoskeletal thigh model with knee and hip joint (6 DoF and six muscles) that despite of the simplifications and our naive model there were good results in the subjects without gait pathologies.

Future work will focuses on the inclusion of electro myography data, more muscles and DoF to achieve to a better understanding of muscle behavior in patients with CP. Anyway the main problem is to record EMGs data for all modeled muscles in clinical practice.

\section{ACKNOWLEDGEMENTS}

The authors would like to thank FLENI Institute for Neurological Research for providing data from patients and healthy subjects and to the National Council of Scientific and Technical Research (CONICET), PICTO 222-2009 (A GENCIA) and PID 6125 (UNER) to provide the funds needed for this research.

\section{REFERENCES}

[1] U. G. Narayanan, "The role of gait analy sis in the orthopaedic management of ambulatory cerebral palsy.," Current opinion in pediatrics, vol. 19, no. 1, pp. 38-43, Feb. 2007.

[2] J. M. Quinby and A. Abraham, "Musculoskeletal problems in cerebral palsy," Current Paediatrics, vol. 15, no. 1, pp. 9-14, Feb. 2005. 
[3] Z. Karim, A. Kranzl, M. Gfoehler, and M. G. Pandy, "Biomechanics of a crouched gait caused by spastic cerebral palsy in children," 8th World Congress on Computional Mechanics, pp. 4-5, 2008.

[4] B. Lofterød, T. Terjesen, I. Skaaret, A.-B. Huse, and R. Jahnsen, "Preoperative gait analysis has a substantial effect on orthopedic decision making in children with cerebral palsy: comparison between clinical evaluation and gait analysis in 60 patients.," Acta orthopaedica, vol. 78, no. 1, pp. 74-80, Feb. 2007.

[5] J. R. Gage and T. F. Novacheck, "An up date on the treatment of gait problems in cerebral palsy.," Journal of Pediatric Orthop aedics. Part B, vol. 10, no. 4, pp. 265-74, Oct. 2001.

[6] M. C. D. M. Filho, R. Yoshida, W. D. S. Carvalho, H. E. Stein, and N. F. Novo, "Are the recommendations from three-dimensional gait analysis associated with better postoperative outcomes in patients with cerebral palsy?,"Gait \& posture, vol. 28, no. 2, pp. 316-22, Aug. 2008.

[7] T. F. Novacheck and J. R. Gage, "Orthopedic management of spasticity in cerebral palsy.," Child's nervous system: ChNS: official journal of the International Society for Pediatric Neurosurgery, vol. 23, no. 9, pp. 1015-31, Sep. 2007.

[8] M. M. van der Krogt, S. L. Delp, and M. H. Schwartz, "How robust is human gait to muscle weakness?," Gait \& posture, vol. 36, no. 1, pp. 113-9, May 2012.

[9] A. S. Arnold, S. S. Blemker, and S. L. Delp, "Evaluation of a Deformable Musculoskeletal Model for Estimating Muscle-Tendon Lengths During Crouch Gait," Annals of Biomedical Engineering, vol. 29, no. 3, pp. 263-274, Mar. 2001.

[10] S. Delp and A. Arnold, "Hamstrings and psoas lengths during normal and crouch gait: Implications for muscle-tendon surgery," Journal of Orthopaedic Research Society, vol. 14, no. 1, pp. 144-151, 1996.

[11] M. M. van der Krogt, C. a M. Doorenbosch, and J. Harlaar, "Muscle length and lengthening velocity in voluntary crouch gait.," Gait \& posture, vol. 26, no. 4, pp. 532-8, Oct. 2007.

[12] A. S. Arnold, M. Q. Liu, M. H. Schwartz, S. Ounpuu, L. S. Dias, and S. L. Delp, "Do the hamstrings operate at increased muscle-tendon lengths and velocities after surgical lengthening?," Journal of Biomechanics, vol. 39, no. 8, pp. 1498-506, Jan. 2006.

[13] J. L. Hicks, M. H. Schwartz, A. S. Arnold, and S. L. Delp, "Crouched postures reduce the capacity of muscles to extend the hip and knee during the single-limb stance phase of gait.," Journal of Biomechan ics, vol. 41, no. 5, pp. 960-7, Jan. 2008.

[14] A. S. Arnold and S. L. Delp, "The role of musculoskeletal models in patient assessment and treatment," Clinics in Developmental Medicine, pp. 163-177, 2004.

[15] A. S. Arnold, M. Q. Liu, M. H. Schwartz, S. Ounpuu, and S. L. Delp, "The role of estimating muscle-tendon lengths and velocities of the hamstrings in the evaluation and treatment of crouch gait.," Gait \& posture, vol. 23, no. 3, pp. 273-81, Apr. 2006.

[16] B. I. Prilutsky and V. M. Zatsiorsky, "Optimization-based models of muscle coordination," Exercise and Sport Sciences Reviews, vol. 30, no. 1, pp. 1-13, 2002.
[17] B. Prilutsky, "Forces of individual cat ankle extensor muscles during locomotion predicted using static optimization," Journal of Biomechanics, vol. 9290, no. 97, 1997.

[18] A. S. Arnold and S. L. Delp, "Computer modeling of gait abnormalities in cerebral palsy: application to treatment planning," Theoretical Issues in Ergonomics Science, vol. 6, no. 3-4, pp. 305-312, May 2005.

[19] H. G. Chambers, "Treatment of functional limitations at the knee in ambulatory children with cerebral palsy.," European Journal of Neurology: the official journal of the European Federation of Neurological Societies, vol. 8, no. 5, pp. 59-74, Nov. 2001.

[20] A. S. Arnold, F. C. Anderson, M. G. Pandy, and S. L. Delp, "Muscular contributions to hip and knee extension during the single limb stance phase of normal gait: a framework for investigating the causes of crouch gait.," Journal of Biomechanics, vol. 38, no. 11, pp. 2181-9, Nov. 2005.

[21] A. Arnold and S. Salinas, "Accuracy of muscle moment arms estimated from MRI-Based musculoskeletal models of the lower extremity," Computer Aided Surgery, vol. 119, pp. 108-119, 2000.

[22] M.M. van der Krogt, C. a M. Doorenbosch, J. G. Becher, and J. Harlaar, "Walking speed modifies spasticity effects in gastrocnemius and soleus in cerebral palsy gait.," Clinical biomechanics (Bristol, Avon), vol. 24, no. 5, pp. 422-8, Jun. 2009.

[23] E. P. Ravera, M. J. Crespo, P. A. Catalfamo, and A. A. Braidot, "Model to estimate hamstrings behavior in cerebral palsy patients: as a pre-surgical clinical diagnosis tool.," Conference proceed ings: Annual International Conference of the IEEE En gin eering in Medicine and Biology Society. IEEE Engineering in Medicine and Biology Society. Conference, vol. 2010, no. 343, pp. 5456-9, Jan. 2010.

[24] J. Perry and J. M. Burnfield, Gait Analysis: Normal and Pathological Function, 2nd ed., SLACK Incorporated, USA, 2010.

[25] R. B. Davis, S. Õunpuu, D. Tyburski, and J. R. Gage, “A gait analysis data collection and reduction technique," Human Movement Science, vol. 10, no. 5, pp. 575-587, Oct. 1991.

[26] M. D. Klein Horsman, H. F. J. M. Koopman, F. C. T. van der Helm, L. P. Prosé, and H. E. J. Veeger, "Morphological muscle and joint parameters for musculoskeletal modelling of the lower extremity.," Clinical biomechanics (Bristol, Avon), vol. 22, no. 2, pp. 239-47, Feb. 2007.

[27] K. R. Kaufman, K. W. An, W. J. Litchy, and E. Y. Chao, "Physiological prediction of muscle forces I. Theoretical formulation.," Neuroscience, vol. 40, no. 3, pp. 781-92, Jan. 1991.

[28] J. Nocedal and S. Wright, Numerical optimization, 2nd ed., Springer, USA, 1999.

[29] A. G. Schache, T. V. Wrigley, R. Baker, and M. G. Pandy, "Biomechanical response to hamstring muscle strain injury.," Gait \& posture, vol. 29, no. 2, pp. 332-8, Feb. 2009.

[30] K. M. Steele, A. Seth, J. L. Hicks, M. S. Schwartz, and S. L. Delp, "Muscle contributions to support and progression during single-limb stance in crouch gait.," Journal of biomechanics, vol. 43, no. 11, pp. 2099-105, Aug. 2010. 